\title{
Effects of vitamin A, C and E, or omega-3 fatty acid supplementation on the level of paraoxonase and arylesterase activity in streptozotocin-induced diabetic rats: an investigation of activities in plasma, and heart and liver homogenates
}

\author{
Mahnaz Zarei ${ }^{1}$, Ms, Shima Fakher ${ }^{1}$, PhD, Seyed Mohammad Bagher Tabei ${ }^{2}$, PhD, Mohammad Hassan Javanbakht ${ }^{1}$, MD, PhD, \\ Hoda Derakhshanian ${ }^{1}$, PhD, Payam Farahbakhsh-Farsi ${ }^{1}$, MD, PhD, Mohammad Reza Sadeghi ${ }^{3}$, PhD, Ebrahim Mostafavi ${ }^{4}$, PhD, \\ Mahmoud Djalali ${ }^{1}$, PhD
}

INTRODUCTION This study was designed and conducted to evaluate the effects of vitamin A, C and E supplementation, and omega-3 fatty acid supplementation on the activity of paraoxonase and arylesterase in an experimental model of diabetes mellitus.

METHODS A total of 64 male Sprague Dawley® rats, each weighing 250 g, were randomly distributed into four groups: (a) normal control; (b) diabetic control; (c) diabetic with vitamin A, C and E supplementation; and (d) diabetic with omega-3 fatty acid supplementation. The animals were anaesthetised after four weeks of intervention, and paraoxonase and arylesterase activity in blood plasma, and liver and heart homogenates were measured.

RESULTS Arylesterase activity in the heart and liver homogenates was significantly lower in the diabetic control group than in the normal control group $(p<0.01)$. Vitamin A, C and E supplementation, and omega-3 fatty acid supplementation significantly increased liver arylesterase activity $(p<0.05)$. No significant change was observed in paraoxonase activity and other investigated factors.

CONCLUSION Vitamin A, C and E, or omega-3 fatty acid supplementation were found to increase liver arylesterase activity in streptozotocin-induced diabetic rats. These supplements may be potential agents for the treatment of diabetes mellitus complications.

Keywords: arylesterase, omega-3 fatty acid, paraoxonase, vitamin C, vitamin $E$

\section{INTRODUCTION}

Diabetes mellitus, one of the most common metabolic disorders in the world, is known to induce oxidant/antioxidant imbalances. The crucial role of reactive oxygen species in the development and exacerbation of diabetes mellitus complications has been studied for several decades. ${ }^{(1,2)}$ Lipid peroxidation is a degenerative process that affects all lipid-containing structures in cells that are under oxidative stress, leading to cytopathological consequences. ${ }^{(3)}$ Although eukaryotic cells possess primary and secondary defences against the deleterious effects of oxidative stress, serious injury can occur when these defence systems are overwhelmed. In such situations, supplementation with antioxidants such as vitamins A, C and E may be beneficial. ${ }^{(4)}$ Vitamin E, a hydrophobic antioxidant found in lipoproteins and cellular membranes, can provide effective protection against lipid peroxyl radicals. ${ }^{(5)}$ Vitamin $\mathrm{C}$ is a hydrophilic antioxidant that can scavenge free radicals; it is likely to act synergistically with vitamin $\mathrm{E}$ by reducing oxidised tocopheroxyl radicals back to tocopherol. ${ }^{(6)}$ Vitamin A, on the other hand, plays an important role in modulating insulin response. ${ }^{(7)}$

Supplementation with omega-3 fatty acids may be useful in preventing diabetes mellitus complications, as long-chain omega-3 fatty acids are incorporated into cell membranes and have anti-inflammatory properties that could prevent diabetes mellitus (omega-3 fatty acids reduce the expression of interleukin-1 beta and human leukocyte antigen class II alleles in activated human monocytes). ${ }^{(8,9)}$ These fatty acids also play a pivotal role in eicosanoid metabolism. There is evidence of abnormal prostaglandin metabolism in children with type 1 diabetes mellitus and anti-inflammatory omega-3 fatty acids (i.e. docosahexaenoic acid and eicosapentaenoic acid) may reduce the risk of disease development. ${ }^{(10)}$ Thus, the present study was designed and performed to evaluate the effects of vitamin A, C and E, or omega-3 fatty acid supplementation on the activity of paraoxonase and arylesterase in liver, heart and blood plasma samples of streptozotocin (STZ)-induced diabetic rats.

\footnotetext{
${ }^{1}$ Department of Cellular and Molecular Nutrition, School of Nutritional Sciences and Dietetics, Tehran University of Medical Sciences, Tehran, ${ }^{2}$ Department of Biochemistry, Shiraz University of Medical Sciences, Shiraz, ${ }^{3}$ Department of Reproductive Endocrinology and Embryology, Avesina Research Institute, ${ }^{4}$ Department of Pathology, Tehran University of Medical Sciences, Tehran, Iran

Correspondence: Dr Mahmoud Djalali, Supervisor, Department of Cellular and Molecular Nutrition, School of Nutritional Sciences and Dietetics, Tehran University of Medical Sciences, Poorsina Street, Enghelab Avenue, Tehran 14155-6446, Iran. mjalali87@yahoo.com
} 
Table I. Paraoxonase activity in the plasma, and heart and liver homogenates of the four groups of rats.

\begin{tabular}{lcccc}
\hline Paraoxonase activity & $\begin{array}{c}\text { Normal } \\
\text { control group } \\
(\mathbf{n}=\mathbf{1 6})\end{array}$ & $\begin{array}{c}\text { Control group } \\
\mathbf{( n = 1 6 )}\end{array}$ & $\begin{array}{c}\text { Vitamin A, C and E } \\
\text { supplementation } \mathbf{( n = 1 6 )}\end{array}$ & $\begin{array}{c}\text { Omega-3 fatty acid } \\
\text { supplementation }(\mathbf{n}=\mathbf{1 6})\end{array}$ \\
\hline In plasma (IU/L) & $29.41 \pm 7.85$ & $27.40 \pm 7.31$ & $29.11 \pm 6.05$ & $28.77 \pm 10.42$ \\
In liver homogenate (IU/mg protein) & $157.78 \pm 101.11$ & $146.58 \pm 52.39$ & $153.90 \pm 25.66$ & $153.93 \pm 16.08$ \\
In heart homogenate (IU/mg protein) & $8.24 \pm 3.37$ & $7.51 \pm 3.20$ & $7.73 \pm 2.60$ & $7.81 \pm 3.93$ \\
\hline
\end{tabular}

Data presented as mean \pm standard deviation. Student's $t$-test was used to compare the values between groups. No significant differences were found between the groups.

Table II. Arylesterase activity in the plasma, and heart and liver homogenates of the four groups of rats.

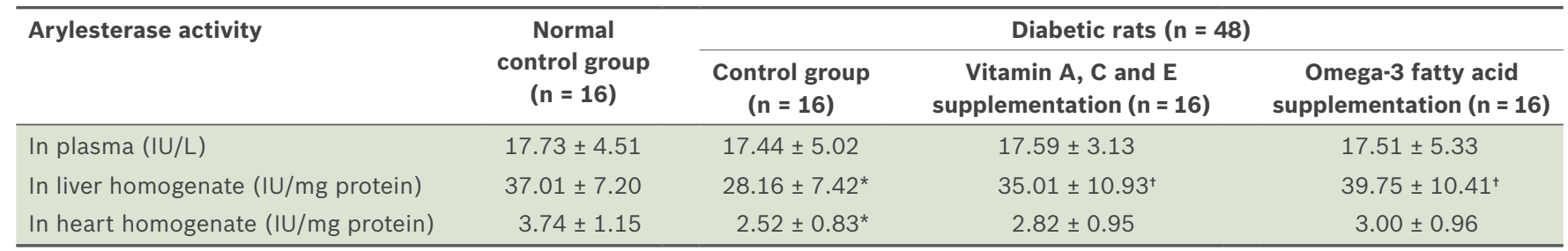

Data presented as mean \pm standard deviation. Student's $t$-test was used to compare the values between groups. ${ }^{*} p<0.01$, compared with the normal control group. $+\mathrm{p}<0.05$, compared with the diabetic control group.

\section{METHODS}

A total of 64 male Sprague Dawley® rats, aged 15 weeks and weighing 250 g each, were used in this study. During the experimental period, the rats had ad libitum access to standard chow and tap water, and were housed in an environment with a temperature of $18^{\circ} \mathrm{C}-23^{\circ} \mathrm{C}$ and a $12: 12$ light-dark cycle. The rats were randomly distributed into four groups of 16 rats each. The four groups were: (a) normal control; (b) diabetic control; (c) diabetic with vitamin A, C and E supplementation; and (d) diabetic with omega-3 fatty acid supplementation.

Diabetes mellitus was induced, via intravascular injection of a single dose of STZ (40 mg/kg body weight), in all animals except those in the normal control group. At one week following the injection, the plasma glucose levels of the rats were checked using the glucose oxidase/p-aminophenazone method of the enzymatic colourimetric test. Rats with a plasma glucose level of more than $300 \mathrm{mg} / \mathrm{dL}$ were considered diabetic. All 48 rats that were injected with STZ had a plasma glucose level of more than $300 \mathrm{mg} / \mathrm{dL}$. The diabetic rats in the vitamin $\mathrm{A}, \mathrm{C}$ and $\mathrm{E}$ supplementation group received vitamin $\mathrm{A}$ $106 \mathrm{mg} / \mathrm{kg}$, vitamin C $200 \mathrm{mg} / \mathrm{kg}$ and vitamin E $250 \mathrm{mg} / \mathrm{kg}$ daily by oral gavage, while diabetic rats in the omega- 3 supplementation group received omega-3 fatty acids $300 \mathrm{mg} / \mathrm{kg}$ daily by oral gavage.

After four weeks of intervention, the rats were anaesthetised and arterial blood samples were collected in tubes containing ethylenediaminetetraacetic acid (EDTA). After the blood samples were centrifuged at 2500 revolutions per minute (rpm) for five minutes, the plasma was separated and the erythrocytes were washed three times in $5 \mathrm{~mL}$ of $9 \%$ sodium chloride $(\mathrm{NaCl})$. Total haemoglobin was measured and the erythrocytes were stored at $-70^{\circ} \mathrm{C}$ until analysis for biochemical factors. Liver and heart tissues were promptly excised from anaesthetised rats and washed with normal saline ( $\mathrm{NaCl} 0.9 \%$ ). The harvested tissues were then dried using filter paper and stored at $-70^{\circ} \mathrm{C}$.
To determine catalase and superoxide dismutase activities, $1: 11(\mathrm{w} / \mathrm{v})$ tissue homogenate was prepared in potassium phosphate buffer ( $\mathrm{pH}$ 7.4) $50 \mathrm{mM}$, potassium chloride $150 \mathrm{mM}$ and EDTA $200 \mathrm{mM}$. The mixture was homogenised using a Potter-Elvehjem Tissue Grinder and centrifuged at 15,000 rpm for 30 minutes. The protein concentration of the homogenate was then determined using the biuret protein assay, with bovine serum albumin as the protein standard. ${ }^{(11)}$ Paraoxonase activity in the liver and heart homogenates, and blood plasma was measured using the rate of paraoxon hydrolysis at $412 \mathrm{~nm}$. As most commercial paraoxon samples contain breakdown products, some of which interfere with the spectrophotometric assay of paraoxonase, it was necessary to purify the paraoxonase prior to use. ${ }^{(12)}$ Arylesterase activity in the liver and heart homogenates, and blood plasma was measured using the rate of phenylacetate hydrolysis at $270 \mathrm{~nm}$.

Data was presented as mean \pm standard deviation. SPSS version 11.5 (SPSS Inc, Chicago, IL, USA) was used for all analyses. Independent sample $t$-test was used to compare the mean between groups. A value of $p<0.05$ was considered to be statistically significant.

\section{RESULTS}

The levels of paraoxonase and arylesterase activity in the different experimental groups are presented in Tables I and II. Paraoxonase activity was lower in the rats in the diabetic control group as compared to the rats in the normal control group. Compared to the diabetic control group, supplementation with vitamins $A$, $\mathrm{C}$ and $\mathrm{E}$, or omega- 3 fatty acids resulted in a modest elevation in paraoxonase activity. However, these differences were not statistically significant. Arylesterase activity was significantly lower in the heart and liver homogenates of the rats in the diabetic control group, as compared to the rats in the normal control group $(p<0.05)$. When compared to the diabetic control group, supplementation with vitamins $\mathrm{A}, \mathrm{C}$ and $\mathrm{E}$, or omega- 3 fatty acids 
resulted in a significant increase in arylesterase activity in the liver homogenate. There was no significant difference in arylesterase activity between the two groups of diabetic rats that received supplementation (vitamins A, C and E, or omega-3 fatty acids).

\section{DISCUSSION}

Based on the data obtained in the present study, which involved rats with STZ-induced diabetes mellitus, arylesterase activity was lower in the heart and liver homogenates of diabetic control rats as compared to that of rats from the normal control group $(p<0.01)$. The results of the present study also showed that after four weeks of vitamin A, C and E supplementation, or omega-3 fatty acid supplementation, arylesterase activity was enhanced in the liver homogenates of the diabetic rats $(p<0.05)$. The level of paraoxonase activity was not found to be significantly different after supplementation with vitamins A, C and E, or omega-3 fatty acids.

Paraoxonase, which has been shown to decrease in diabetic subjects, may be a predictor of complications of diabetes mellitus. ${ }^{(13)}$ Both Kang et al and Sampson et al reported a decrease in paraoxonase activity in patients with diabetes mellitus. ${ }^{(14,15)}$ This may be due to the inactivation of paraoxonase via glycation, a decrease in its gene expression or the inhibition of high-density lipoprotein synthesis or secretion, which is reported to be associated with serum paraoxonase. ${ }^{(14,16)}$ In the present study, we did not observe any significant changes in paraoxonase activity. A possible reason is the relatively short intervention period of four weeks, which may not have been sufficient to detect the effect of supplementation. This is one of the limitations of our study.

It has also been shown that glucose autoxidation, protein glycation and the production of advanced glycation end products are the main mechanisms by which oxygen free radicals are produced in patients with diabetes mellitus. ${ }^{(17,18)}$ In diabetic patients, beta-oxidation of fatty acids increases as a result of insufficient insulin. This, in turn, results in the accumulation of hydrogen peroxide in tissues, leading to enzyme inactivation via glycation. Due to the higher concentration of hydrogen peroxide in the tissue than in the blood, enzyme inactivation via glycation is more pronounced in the tissues. ${ }^{(19)}$

The liver is the main organ responsible for synthesising paraoxonase and arylesterase; thus, inhibiting oxidative damage with antioxidant (i.e. vitamins $\mathrm{A}, \mathrm{C}$ and $\mathrm{E}$, and omega-3 fatty acids) supplementation causes significant changes in the activity of these two key enzymes in the liver homogenate. As vitamins $\mathrm{A}, \mathrm{C}$ and $\mathrm{E}$ have different sites of action and different mechanisms to prevent oxidative damage, these vitamins are expected to be more effective when used in combination than individually. ${ }^{(20)}$ The ability of ascorbic acid (vitamin C) to reduce alpha-tocopheroxyl radicals to alpha-tocopherol, inhibiting the resulting oxidative damage, has been demonstrated in both in vitro and in vivo studies. ${ }^{(21,22)}$ Vitamin $\mathrm{E}$ may help regulate intracellular magnesium levels, which diminish with increased oxygen free radical production. While vitamin $\mathrm{C}$ scavenges reactive oxygen species in the hydrophilic milieu, alpha- tocopherol and vitamin A inhibit lipid oxidation by free radical chain reaction in the hydrophobic domains of the bilayer. ${ }^{(23,24)}$ Supplementation with vitamins $\mathrm{C}$ and $\mathrm{E}$ has been reported to increase antioxidant enzyme activity. ${ }^{(25)}$ Similarly, omega-3 fatty acid supplementation might also increase antioxidant enzyme activity. ${ }^{(26)}$ Although the underlying mechanism responsible for the beneficial effects of omega-3 fatty acids on oxidative status has not been fully understood, several studies indicate that omega-3 fatty acids may play a pivotal role in decreasing the complications of diabetes mellitus. ${ }^{(27,28)}$

In conclusion, the results of the present study indicated that four weeks of supplementation with vitamins $A, C$ and $E$, or omega- 3 fatty acid can improve the activity of arylesterase in the liver. It may do this by decreasing free radical production and/or inhibiting enzyme destruction. Further study is needed to shed light on the specific mechanisms by which such supplementation improves antioxidant enzyme activity.

\section{ACKNOWLEDGEMENT}

This study was conducted using a grant from the Tehran University of Medical Sciences, Iran.

\section{REFERENCES}

1. Maritim AC, Sanders RA, Watkins JB 3rd. Diabetes, oxidative stress, and antioxidants: a review. J Biochem Mol Toxicol 2003; 17:24-38.

2. Rahimi R, Nikfar S, Larijani B, Abdollahi M. A review on the role of antioxidants in the management of diabetes and its complications. Biomed Pharmacother 2005; 59:365-73.

3. Uchida K. Future of toxicology--lipid peroxidation in the future: from biomarker to etiology. Chem Res Toxicol 2007; 20:3-5.

4. Seven A, Guzel S, Seymen O, et al. Effects of vitamin E supplementation on oxidative stress in streptozotocin induced diabetic rats: investigation of liver and plasma. Yonsei Med J 2004; 45:703-10.

5. Niki E, Noguchi N. Dynamics of antioxidant action of vitamin E. Acc Chem Res 2004; 37:45-51.

6. Liu D, Shi J, Colina Ibarra A, Kakuda Y, Xue SJ. The scavenging capacity and synergistic effects of lycopene, vitamin $\mathrm{E}$, vitamin $\mathrm{C}$, and $\beta$-carotene mixtures on the DPPH free radical. LWT Food Sci Technol 2008; 41:1344-9.

7. Berry DC, Noy N. Signaling by vitamin A and retinol-binding protein in regulation of insulin responses and lipid homeostasis. Biochim Biophys Acta $2012 ; 1821: 168-76$.

8. Norris JM, Yin X, Lamb MM, et al. Omega-3 polyunsaturated fatty acid intake and islet autoimmunity in children at increased risk for type 1 diabetes. JAMA 2007; 298:1420-8.

9. Calder PC. Polyunsaturated fatty acids and inflammatory processes: New twists in an old tale. Biochimie 2009; 91:791-5.

10. Litherland S, Xie XT, Hutson AD, et al. Aberrant prostaglandin synthase 2 expression defines an antigen-presenting cell defect for insulin-dependent diabetes mellitus. J Clin Invest 1999; 104:515-23.

11. Olson BJ, Markwell J. Assays for determination of protein concentration. Curr Protoc Protein Sci 2007; Chapter 3:Unit3.4.

12. Camps J, Marsillach J, Joven J. The paraoxonases: role in human diseases and methodological difficulties in measurement. Crit Rev Clin Lab Sci 2009; 46:83-106.

13. Ikeda $Y$, Inoue $M$, Suehiro $T$, et al. Low human paraoxonase predicts cardiovascular events in Japanese patients with type 2 diabetes. Acta Diabetol 2009; 46:239-42.

14. Kang KA, Chae S, Koh YS, et al. Protective effect of puerariae radix on oxidative stress induced by hydrogen peroxide and streptozotocin. Biol Pharm Bull 2005; 28:1154-60.

15. Sampson MJ, Braschi S, Willis G, Astley SB. Paraoxonase-1 (PON-1) genotype and activity and in vivo oxidized plasma low-density lipoprotein in Type II diabetes. Clin Sci (Lond) 2005; 109:189-97.

16. Ferré N, Camps J, Prats E, et al. Serum paraoxonase activity: a new additional test for the improved evaluation of chronic liver damage. Clin 
Chem 2002; 48:261-8

17. Miyazawa T, Nakagawa K, Shimasaki S, Nagai R. Lipid glycation and protein glycation in diabetes and atherosclerosis. Amino Acids 2012; 42:1163-70.

18. Moheimani F, Morgan PE, van Reyk DM, Davies MJ. Deleterious effects of reactive aldehydes and glycated proteins on macrophage proteasomal function: possible links between diabetes and atherosclerosis. Biochim Biophys Acta 2010; 1802:561-71.

19. Giacco F, Brownlee M. Oxidative stress and diabetic complications. Circ Res 2010; 107:1058-70.

20. Bürzle M, Hediger MA. Functional and physiological role of vitamin C transporters. Curr Top Membr 2012; 70:357-75.

21. Yeum KJ, Beretta G, Krinsky NI, Russell RM, Aldini G. Synergistic interactions of antioxidant nutrients in a biological model system. Nutrition 2009; 25:839-46.

22. Niki E. Assessment of antioxidant capacity in vitro and in vivo. Free Radic Biol Med 2010; 49:503-15
23. Atkinson J, Harroun T, Wassall SR, Stillwell W, Katsaras J. The location and behavior of alpha- tocopherol in membranes. Mol Nutr Food Res 2010; 54:641-51.

24. Zago MP, Oteiza PI. The antioxidant properties of zinc: interactions with iron and antioxidants. Free Radic Biol Med 2001; 31:266-74.

25. Day R, Lal SS. Supplementation effects of vitamin C and vitamin E on oxidative stress in post menopausal diabetic women. J Appl Res 2012; 12:108-11.

26. Garrel C, Alessandri JM, Guesnet P, Al-Gubory KH. Omega-3 fatty acids enhance mitochondrial superoxide dismutase activity in rat organs during post-natal development. Int J Biochem Cell Biol 2012; 44:123-31.

27. McEwen B, Morel-Kopp MC, Tofler G, Ward C. Effect of omega-3 fish oil on cardiovascular risk in diabetes. Diabetes Educ 2010; 36:565-84.

28. Stirban A, Nandrean S, Götting C, et al. Effects of n-3 fatty acids on macro- and microvascular function in subjects with type 2 diabetes mellitus. Am J Clin Nutr 2010; 91:808-13. 